\title{
Near Surface Effects on the Flagellar Propulsion of Soft Robotic Sperms
}

\author{
Islam S. M. Khalil*, Mohamed Hafez ${ }^{*}$, Anke Klingner ${ }^{\ddagger}$, Stefano Scheggi ${ }^{\dagger}$, Barbara Adel ${ }^{\natural}$, and Sarthak Misra ${ }^{\dagger \S}$
}

\begin{abstract}
In this work, we investigate the near surface effects on the flagellar propulsion of externally actuated soft robotic sperms. A group of $250-\mu m$-long robotic sperms are fabricated using electrospinning, and the influence of a nearby wall on their flagellar propulsion is modeled and characterized inside a fluidic chip with channels of varying width. Our experimental results show that the swimming speed of the robotic sperm decreases by a factor of 2 when its distance to a nearby surface is decreased by $\mathbf{5 0} \%$, at frequency and precision angle of $5 \mathrm{~Hz}$ and $15^{\circ}$, respectively. We also show that the reduction in swimming speed can be mitigated by adapting the beating frequency and the precision angle of the tail and head of the robotic sperm during flagellar propulsion. We also demonstrate point-to-point closed-loop control along a reference trajectory inside a channel of varying width and achieve maximum steady-state error of $5.6 \mu \mathrm{m}$.
\end{abstract}

\section{INTRODUCTION}

Externally actuated biologically inspired microrobots offer promise for a variety of nanotechnology [1], [2] and biomedical [3]-[5] applications. The external actuation enables these microrobots to meet the continuous demand for miniaturization, whereas their natural design allows for locomotion in low Reynolds numbers [6]-[9]. Drug delivery [10], diagnostics [11], cell manipulation and sorting [12], biopsy, and precision nanosurgery [5] have been demonstrated using the mentioned approach. Recently, Fernández de Ávila et al. have demonstrated in vivo antibiotic delivery in the gastric media of a mouse model using drug-loaded magnesium micromotors [13]. Ahmed et al. have also demonstrated effective maneuverability of a hybrid soft microswimmer using a combination of acoustic and magnetic fields [14]. Felfoul et al. have also transported drug-loaded nanoliposomes into hypoxic regions of a tumour using biological microrobots. In these biomedical applications, it is likely that the microrobots will be influenced by near-surface effects such as the background flows and surface interactions. These effects will have significant influence on soft microrobots that undergo controlled deformations to break timereversal symmetry [15], [16]. For instance, elasto-magnetic

This work was supported by the Science and Technology Development Fund in Egypt (No. 23016) and the DAAD-BMBF funding project.

*Department of Mechatronics Engineering, $\ddagger$ Department of Physics, Faculty of ${ }^{b}$ Pharmacy and Biotechnology, the German University in Cairo, New Cairo City 11835, Egypt.

${ }^{\dagger}$ Department of Biomechanical Engineering, MIRA-Institute for Biomedical Technology and Technical Medicine, University of Twente, Enschede 7500 AE, The Netherlands.

$\S$ Department of Biomedical Engineering, University of Groningen and University Medical Centre Groningen, Groningen 9713 AV, The Netherlands.
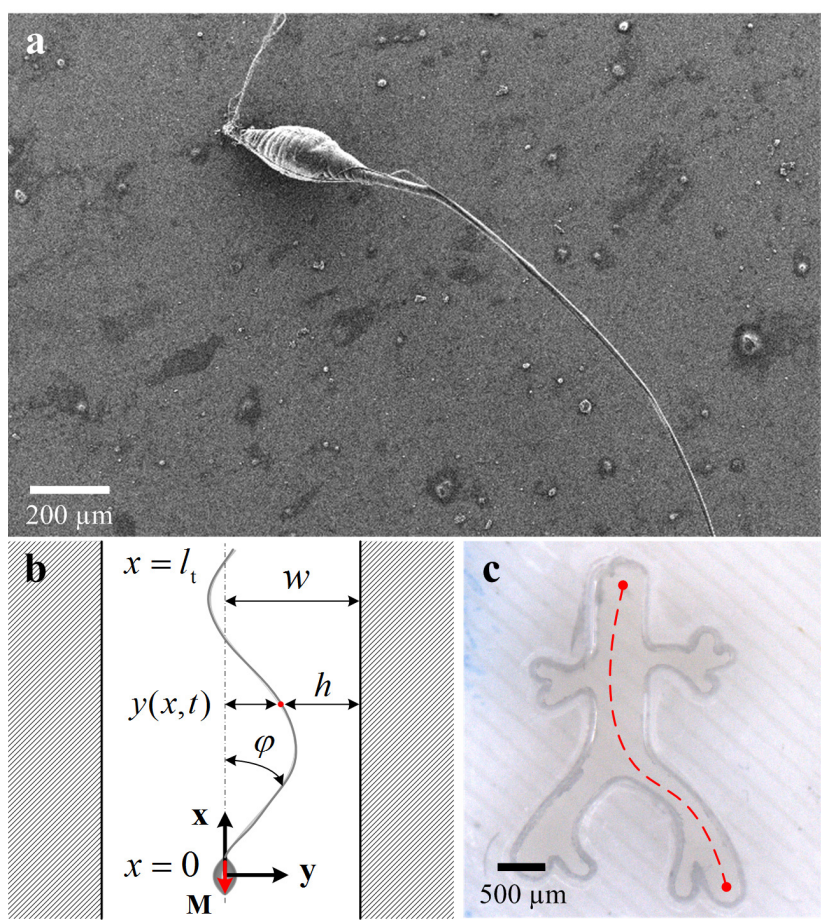

Fig. 1. The influence of a nearby surface on the planar flagellar propulsion of soft robotic sperms is studied. (a) The robotic sperm consists of an elliptical head and an ultra-thin flexible tail [19]. Magnetic particles are embedded into the polymer matrix of the head to provide magnetization (M) and enable directional control. (b) The robots, with tail length $l_{\mathrm{t}}$, are contained inside a fluidic chip and the influence of the proximity $(h)$ to a surface on the flagellar propulsion is characterized. (c) Point-to-point control of the robotic sperm is achieved in a channel with a bifurcation to study the influence of the proximity to a nearby surface on the flagellar swim. The dashed red trajectory indicates the path of the soft robotic sperm.

microrobots [17] swim using the propagation of planar or three-dimensional travelling waves, and the propulsive forces imparted to the background medium enable locomotion. In this work, we study the influence of a nearby surface on the planar flagellar propulsion of a soft robotic sperm [Fig. 1(a)], and achieve the following: (1) Characterization of the swimming speed during flagellar propulsion for a varying proximity to a nearby surface inside a fluidic chip [Fig. 1(b)] (2) Investigation of the beating frequency of the tail and the precision angle of the head on the swimming speed for varying proximities. (3) Closed-loop control inside a fluidic chip of varying width to demonstrate the capability of the robotic sperm to maintain a fixed swimming speed. The remainder of this paper is organized as follows: Section II 
provides a physical picture for the planar flagellar propulsion and the influence of a nearby surface on the propulsive forces imparted to the fluid. Frequency response characterization for varying proximities to a channel wall is included in Section II. Section III provides our closed-loop control results. Finally, Section IV concludes and provides directions for future work.

\section{Modeling of the Soft Robotic Sperm}

Our soft robotic sperm consists of an elliptical magnetic head (with minor diameter $2 r_{\mathrm{h}}$ and major diameter $l_{\mathrm{h}}$ ) that is rigidly connected to an ultra-thin flexible tail with bending stiffness $A$, length $l_{\mathrm{t}}$, and diameter $2 r_{\mathrm{t}}$. The magnetic head has an average magnetic moment $\mathbf{M}$ and is subjected to periodic magnetic field $\mathbf{B}$, while immersed in a viscous medium with viscosity $\mu$ inside a narrow channel. The medium gap between the robot and a nearby surface has width $w$. Magnetization of the robot is oriented along the long axis. This magnetic moment generates a bending wave along the ultra-thin flexible tail which is governed by

$$
A \frac{\partial^{4}}{\partial x^{4}} y(x, t)+c_{\mathrm{n}}(h) \frac{\partial}{\partial t} y(x, t)=0,
$$

where $y(x, t)$ describes the flexible tail, relative to a fixed frame of reference. Further, $c_{\mathrm{n}}(h)$ is the normal drag coefficient and is given by [18]

$$
c_{\mathrm{n}}(h)=\frac{4 \pi \mu}{\log \left(\frac{2 h}{r_{\mathrm{t}}}\right)-0.301} \text { and } c_{\mathrm{t}}(h)=\frac{c_{\mathrm{n}}(h)}{2} .
$$

In (2), $h=w-|y(x, t)|$ is the distance between the tail and the nearest surface and $c_{\mathrm{t}}(h)$ is the tangential drag coefficient. The drag coefficient is influenced by the proximity to a nearby surface, as shown in Fig 2. The flexible tail is free from external forces and torques at the right boundary $\left(x=l_{\mathrm{t}}\right)$, hence $\left.\frac{\partial^{2} y}{\partial x^{2}}\right|_{l_{\mathrm{t}}}=0$ and $\left.\frac{\partial^{3} y}{\partial x^{3}}\right|_{\mathrm{t}_{\mathrm{t}}}=0$. The influence of the periodic magnetic field is included at the point of attachment between the magnetic head and the flexible tail, hence $y(0, t)=0$ and $\left.\frac{\partial y}{\partial x}\right|_{0}=\epsilon \sin (\omega t)$, where $\epsilon$ and $\omega$ are the amplitude and frequency of oscillation of the periodic magnetic field, respectively.

We solve (1) numerically for $N_{x}+1$ elements along $\mathrm{x}$-axis of the flexible tail. The deformation of the tail $y(x, t)$ is represented as a vector $\left[\begin{array}{llll}\widetilde{y}_{1} & \widetilde{y}_{2} & \ldots & \widetilde{y}_{N_{x}+1}\end{array}\right]$ and $y(x, t+\Delta t)$ as $\left[\begin{array}{llll}y_{1} & y_{2} & \ldots & y_{N_{x}+1}\end{array}\right]$. The boundary conditions provide $\widetilde{y}_{1}=y_{1}=0, \widetilde{y}_{2}=|\mathbf{B}| \sin (\omega t)$, and $y_{2}=|\mathbf{B}| \sin (\omega(t+\Delta t))$. The magnitude of the magnetic field $(|\mathbf{B}|)$ is proportional to the precision angle using, $|\mathbf{B}|=\Delta x \tan \varphi$. Finally, the derivatives $\frac{\partial y}{\partial x}$ and $\frac{\partial^{2} y}{\partial x^{2}}$ are approximated using

$$
\left.\frac{\partial y}{\partial x}\right|_{i}=\frac{y_{i}-y_{i-1}}{\Delta x} \text { and }\left.\frac{\partial^{2} y}{\partial x^{2}}\right|_{i}=\frac{y_{i}-2 y_{i-1}+y_{i-2}}{\Delta x^{2}} .
$$

The third-order derivative is calculated using

$$
\left.\frac{\partial^{3} y}{\partial x^{3}}\right|_{i}=\frac{y_{i}-3 y_{i-1}+3 y_{i-2}-y_{i-3}}{\Delta x^{3}} .
$$

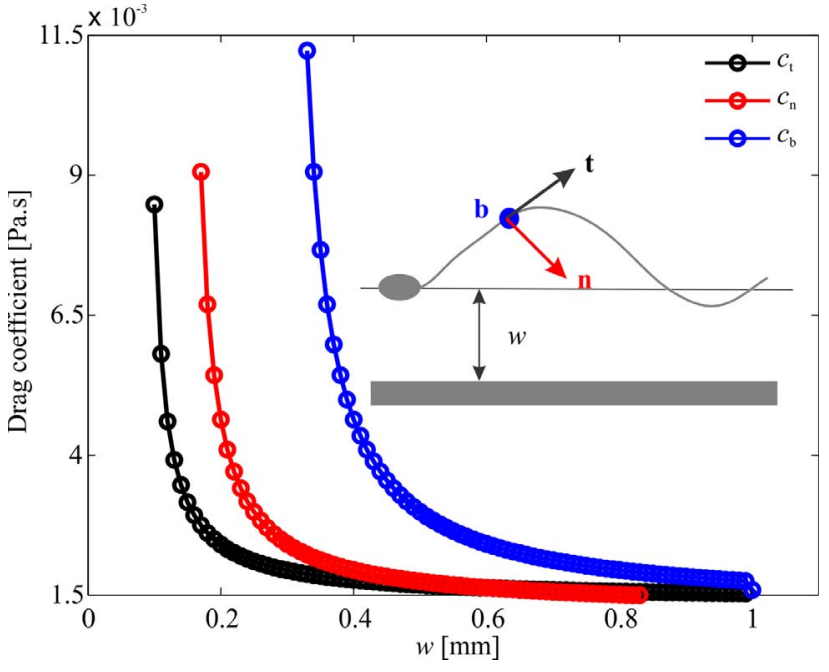

Fig. 2. The influence of proximity to a nearby surface $(w)$ on the tangential $\left(c_{\mathrm{t}}\right)$, normal $\left(c_{\mathrm{n}}\right)$, and bi-normal $\left(c_{\mathrm{b}}\right)$ drag coefficient is calculated using (2). The drag coefficients are calculated for $250-\mu \mathrm{m}$-long robotic sperm with tail diameter of $10 \mu \mathrm{m}$ and in a medium with viscosity of 0.95 Pa.s. The difference between parallel and normal drag (affected by the distance to a channel wall) along the tail of the robotic sperm results in net propulsion.

Finally, the fourth-order derivative is given by

$$
\begin{aligned}
\left.\frac{\partial^{4} y}{\partial x^{4}}\right|_{i} & =\frac{y_{i}-4 y_{i-1}+6 y_{i-2}-4 y_{i-3}+y_{i-4}}{\Delta x^{4}}, \\
& =-\frac{c_{\mathrm{n}}(h)\left(y_{i-2}-\widetilde{y}_{i-2}\right)}{A_{i-2} \Delta t} .
\end{aligned}
$$

Equations (3)-(6) provide a system of $N_{x}-1$ equations for $N_{x}-1$ unknowns, which can be represented as, $\mathbf{S n}=\mathbf{V}$, where matrix $\mathbf{S}$ is given by

$$
\mathbf{S}=\left(\begin{array}{cccccc}
6-f_{3} & -4 & 1 & 0 & \ldots & 0 \\
-4 & 6-f_{4} & -4 & 1 & \ldots & \vdots \\
1 & -4 & 6-f_{5} & -4 & \ddots & 0 \\
0 & 1 & -4 & \ddots & -4 & 1 \\
\vdots & \vdots & \ddots & -3 & 3-f_{N_{x}} & -1 \\
0 & 0 & \vdots & 1 & -2 & 1
\end{array}\right)
$$

In (7), $f_{i}$ is calculated using

$$
f_{i}=\frac{c_{\mathrm{n}}\left(h\left(\widetilde{y}_{i}\right)\right) \Delta x^{4}}{A \Delta t}
$$

The vectors $\mathbf{V}$ and $\mathbf{n}$ are given by

$$
\mathbf{V}=\left(\begin{array}{c}
4 y_{2}-y_{1}-f_{3} \widetilde{y}_{3} \\
-y_{2}-f_{4} \widetilde{y}_{4} \\
-f_{5} \widetilde{y}_{5} \\
\vdots \\
-f_{N_{x}} \widetilde{y}_{N_{x}}
\end{array}\right) \text { and } \mathbf{n}=\left(\begin{array}{c}
y_{3} \\
y_{4} \\
y_{5} \\
\vdots \\
y_{N_{x}+1}
\end{array}\right)
$$



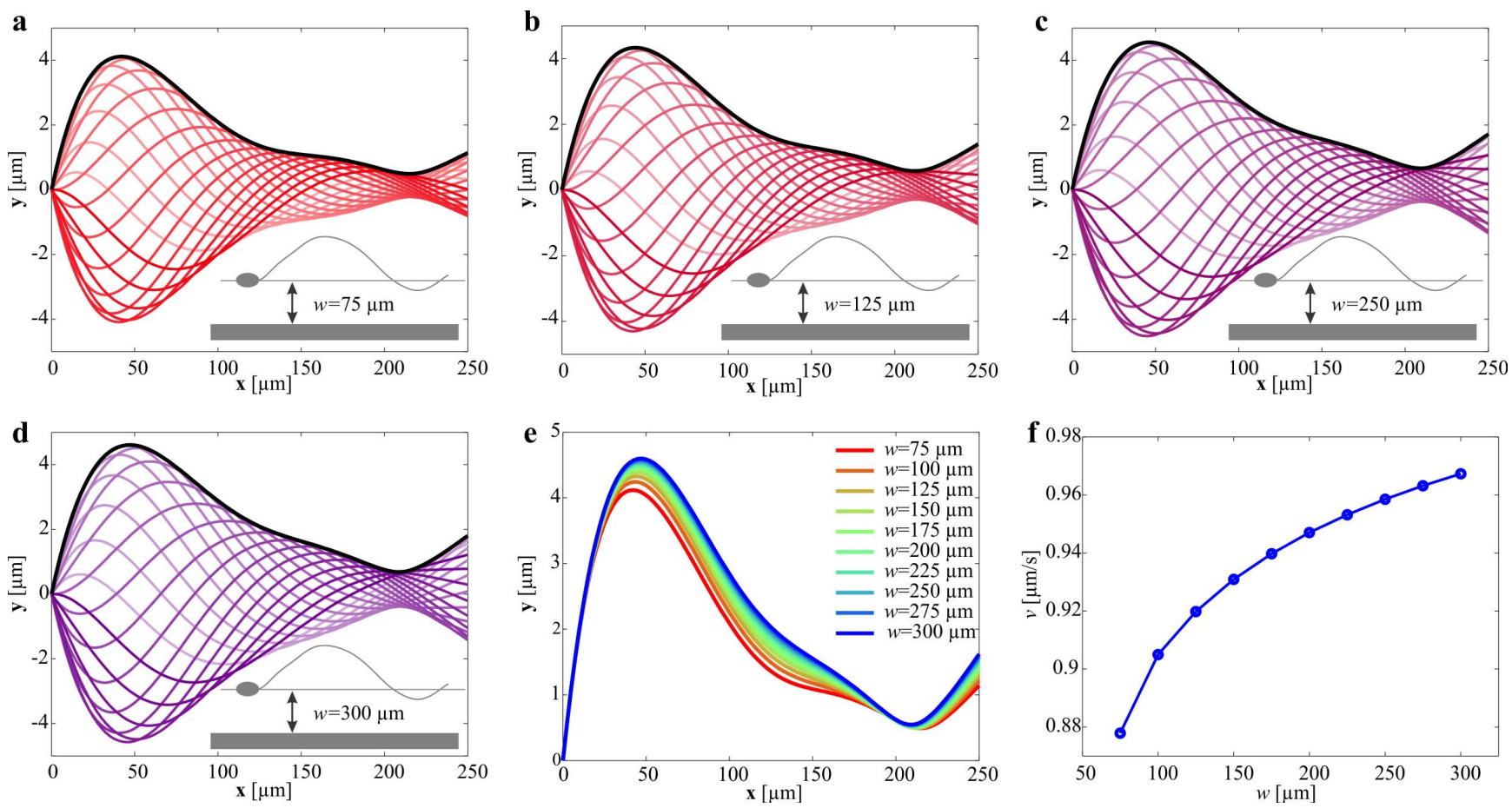

Fig. 3. A nearby surface influences the deformation of the flexible tail and the swimming speed of a robotic sperm. The thick black curve represents the first deformation and the lighter curves represent deformation for representative interval of a complete period. (a) Tail deformation for a complete period over equal intervals at frequency of $1 \mathrm{~Hz}$, for $w=75 \mu \mathrm{m}$. (b) Tail deformation for $f=1 \mathrm{~Hz}$, for $w=125 \mu \mathrm{m}$. (c) Tail deformation for $f=1 \mathrm{~Hz}$, for $w=250 \mu \mathrm{m}$. (d) Tail deformation for $f=1 \mathrm{~Hz}$, for $w=300 \mu \mathrm{m}$. (e) Maximum deformation during a complete period are calculated for each proximity to a nearby surface. (f) The swimming speed of the robotic sperm is calculated for $h=75 \mu \mathrm{m}$ to $h=300 \mu \mathrm{m}$. The drag coefficients are calculated for $250-\mu \mathrm{m}$-long robotic sperm with tail diameter of $10 \mu \mathrm{m}$ and in a medium with viscosity of 1 Pa.s.

The total thrust force of all segments moving with speed $V_{x}$ for small tail deformation is balanced with the drag force on the head as follows:

$\int_{0}^{l_{\mathrm{t}}} \frac{c_{\mathrm{n}}(h)}{2}\left(\frac{\mathrm{d} y}{\mathrm{~d} t} \frac{\mathrm{d} y}{\mathrm{~d} x}-V_{x}\left(1+\left(\frac{\mathrm{d} y}{\mathrm{~d} x}\right)^{2}\right)\right) \mathrm{d} x=6 \pi \mu r_{\mathrm{h}} V_{x}$.

We solve (1)-(10) numerically for a $250-\mu \mathrm{m}$-long robotic sperm with head minor diameter of $30 \mu \mathrm{m}$ and tail radius of $5 \mu \mathrm{m}$, in a viscous medium with viscosity of 0.95 Pa.s. The applied magnetic field is allowed to oscillate within a frequency range of $1 \mathrm{~Hz}$ to $10 \mathrm{~Hz}$ and with precision angle $\varphi$ [Fig. 1(b)]. The deformation of the flexible tail and the forward swimming speed are determined for proximities of $75 \mu \mathrm{m}$ to $300 \mu \mathrm{m}$ with a step of $50 \mu \mathrm{m}$. Figs. 3(a), 3(b), 3(c), and 3(d) show the deformation of the elastic tail of the robotic sperm for proximities of $75 \mu \mathrm{m}, 125 \mu \mathrm{m}, 250 \mu \mathrm{m}$, and $300 \mu \mathrm{m}$, respectively. The deformation is calculated for a complete period over equal intervals at $f=1 \mathrm{~Hz}$. We observe that the amplitude of the propagating wave along the flexible tail increases with the proximity to a nearby surface, as shown in Fig. 3(e). Net propulsion is achieved mainly by the difference between parallel and normal drag along the flexible tail [18]. These drag coefficients are monotonically decreasing with the distance to a nearby surface, as shown in Fig. 2. Therefore, this proximity influences the deformation of the tail, and as a consequence, the swimming speed of the robotic sperm is also affected [Fig. 3(f)].

In another set of simulation results, the deformation of the flexible tail and the swimming speed of the robotic sperm are calculated for actuation frequencies of $1 \mathrm{~Hz}$ to $10 \mathrm{~Hz}$ and the minimum and maximum proximities to a surface, as shown in Fig. 4. Figs. 4(a) and 4(b) show the maximum deformation of the tail for $w=125 \mu \mathrm{m}$ and $w=250 \mu \mathrm{m}$, respectively. The difference between the forward swimming speeds for $w=125 \mu \mathrm{m}$ and $w=250$ $\mu \mathrm{m}$ is negligible, at relatively low actuation frequencies. The influence of the proximity to a nearby surface becomes increasingly effective with the actuation frequency, as shown in Fig. 4(c). Our model predicts a decrease in swimming speed of $0.6 \%$ at $f=1 \mathrm{~Hz}$ and $2.7 \%$ at $f=10 \mathrm{~Hz}$ for $w=125 \mu \mathrm{m}$ and $w=250 \mu \mathrm{m}$, respectively. Therefore, the influence of a channel wall on the swimming speed can be mitigated at relatively low actuation frequencies. Similarly to the actuation frequency, the influence of the precision angle $(\varphi)$ of the head of the robotic sperm is relatively low. Figs. 5(a) and 5(b) show the deformation of the tail for $\varphi=3^{\circ}$ to $\varphi=15^{\circ}$ for $w=125 \mu \mathrm{m}$ and $w=250 \mu \mathrm{m}$, respectively. The swimming speed is decreased by $3.5 \%$ and $3.9 \%$ when swimming with $\varphi=3^{\circ}$ and $\varphi=15^{\circ}$, for $w=125 \mu \mathrm{m}$ and $w=250 \mu \mathrm{m}$, respectively [Fig. 5(c)]. We use the theoretical predictions of our model to control the robotic sperms inside a fluidic channel of varying width. 

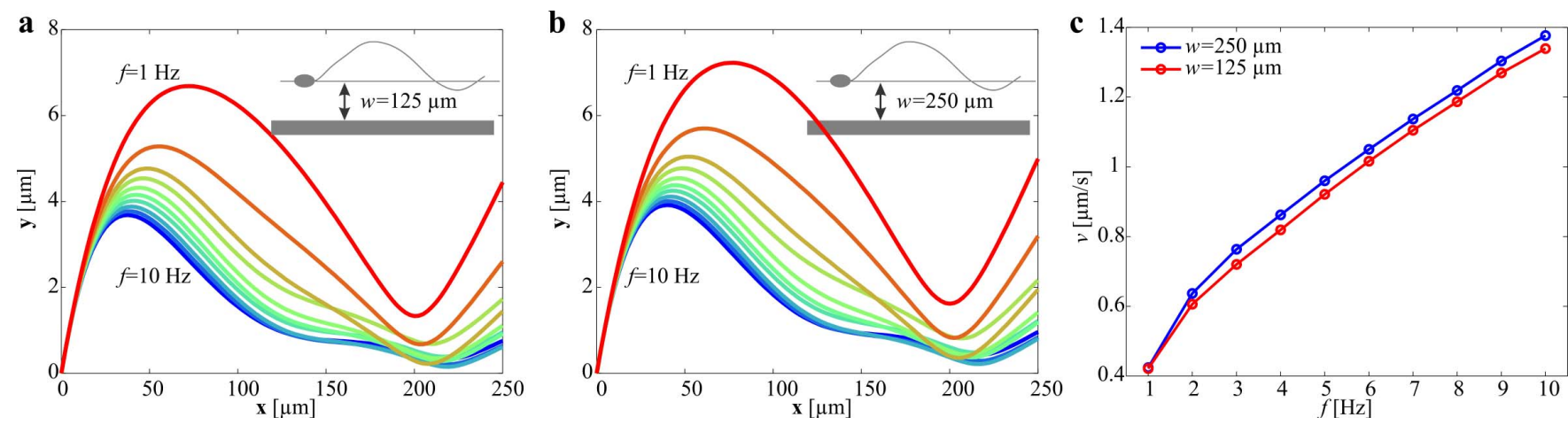

Fig. 4. The influence of a nearby surface and the actuation frequency on the deformation of the tail and the swimming speed of the robotic sperm is analyzed. (a) Maximum tail deformation is calculated for actuation frequencies of $1 \mathrm{~Hz}$ to $10 \mathrm{~Hz}$ when the robotic sperm is at $125 \mu \mathrm{m}$ from a nearby surface. (b) Maximum tail deformation is calculated for actuation frequencies of $1 \mathrm{~Hz}$ to $10 \mathrm{~Hz}$ for $w=250 \mu \mathrm{m}$. (c) The swimming speed of the robotic sperm is calculated for $w=125 \mu \mathrm{m}$ and $w=250 \mu \mathrm{m}$. The deformations and speeds are calculated for 250- $\mu \mathrm{m}$-long robotic sperm with tail diameter of $10 \mu \mathrm{m}$ and in a medium with viscosity of 1 Pa.s.
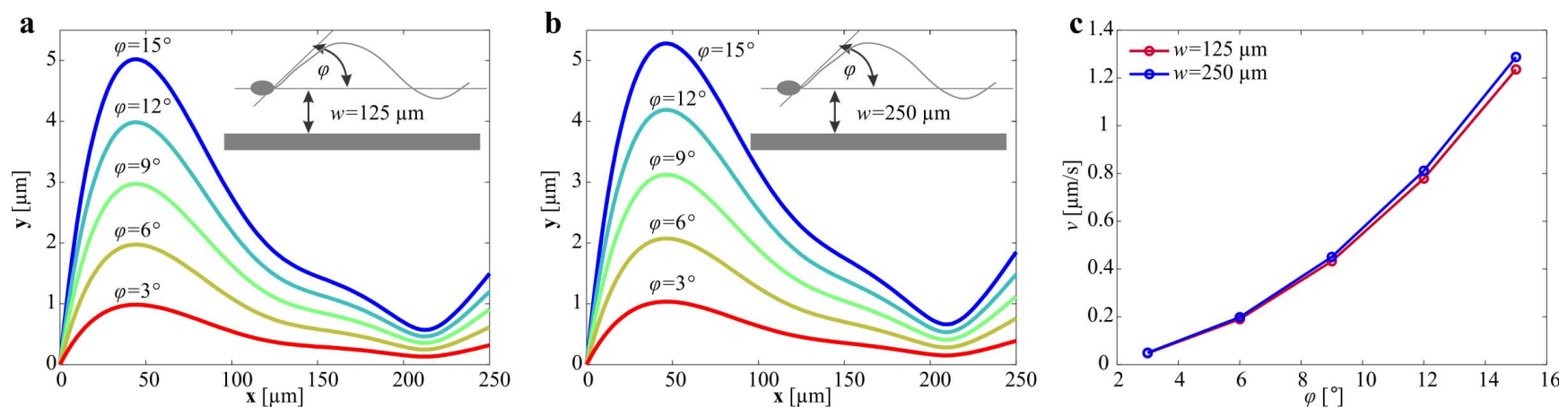

Fig. 5. The influence of a nearby surface and the actuation frequency on the deformation of the tail and the swimming speed of the robotic sperm is analyzed. (a) Maximum tail deformation is calculated for actuation frequencies of $1 \mathrm{~Hz}$ to $10 \mathrm{~Hz}$ when the robotic sperm is at $125 \mu \mathrm{m}$ from a nearby surface. (b) Maximum tail deformation is calculated for actuation frequencies of $1 \mathrm{~Hz}$ to $10 \mathrm{~Hz}$ when the robotic sperm is at $250 \mu \mathrm{m}$ from a nearby surface. (c) The swimming speed of the robotic sperm is calculated for $w=125 \mu \mathrm{m}$ and $w=250 \mu \mathrm{m}$. The deformations and speeds are calculated for $250-\mu \mathrm{m}$-long robotic sperm with tail diameter of $10 \mu \mathrm{m}$ and in a medium with viscosity of 1 Pa.s.

\section{Closed-Loop Control of Robotic Sperms}

Robotic sperms are fabricated and their flagellar propulsion is studied inside a fluidic channel of varying width.

\section{A. Sample Preparation and Experimental Setup}

The robotic sperms are fabricated based on our previous work [19] using electrospinning. All samples used in our study are fabricated by pumping a solution of polystyrene in dimethylformamide (DMF) and magnetic particles with maximum diameter of $30 \mu \mathrm{m}$. The polymer concentration is $25 \mathrm{wt} \%$ in DMF, and the weight ratio of iron:polysterene is $1: 2$. Beaded-fibers are produced by applying a high electric potential of $25 \mathrm{kV}$ at a distance of $10 \mathrm{~cm}$ between the syringe pump and a collector at flow rate of $20 \mu \mathrm{l} / \mathrm{min}$. The dimensions of the robotic sperms are determined using scanning electron microscopy images [Fig. 1(a)] and the average tail length $\left(l_{\mathrm{t}}=250 \mu \mathrm{m}\right)$, head diameter $\left(2 r_{\mathrm{h}}=30 \mu \mathrm{m}\right)$, and tail diameter $2 r_{\mathrm{t}}=10 \mu \mathrm{m}$ are entered to our model (1)-(10). The robotic sperms are contained inside a fluidic chip with depth of $2 \mathrm{~mm}$ and are allowed to swim along channels of varying width. The wider channel bifurcates into narrower channels to examine the influence of the channel wall on the exerted drag on the robotic sperm. The chip is fixed in the common center of 4 identical electromagnetic coils with orthogonal configuration [20]. Each coil has inner- and outer-diameter of $20 \mathrm{~mm}$ and $40 \mathrm{~mm}$, respectively, with 1700 turns. These coils are powered independently to generate a homogenous magnetic field along the direction of motion with sinusoidally varying orthogonal component.

\section{B. Closed-Loop Control Inside a Channel of Varying Width}

Our closed-loop control strategy is based on orienting uniform magnetic fields towards waypoints along a reference path [21]. A sinusoidally varying orthogonal component is applied once the long axis of the robot is aligned towards the nearest waypoint to achieve flagellar propulsion. We examine the motion of the robotic sperm along a channel [Fig. 1(c)] with width of $500 \mu \mathrm{m}$ that bifurcates into two channels with width of $250 \mu \mathrm{m}$. A reference path is provided to enable the robotic sperm to swim along the wide and narrow channels. Fig. 6(a) shows a representative closed-loop control trial of a $250-\mu \mathrm{m}$-long robotic sperm at actuation frequency of $10 \mathrm{~Hz}$. The robotic sperm reaches at the bifurcation point at $t=100$ seconds, as shown in Fig. 6(b). We observe that the average 

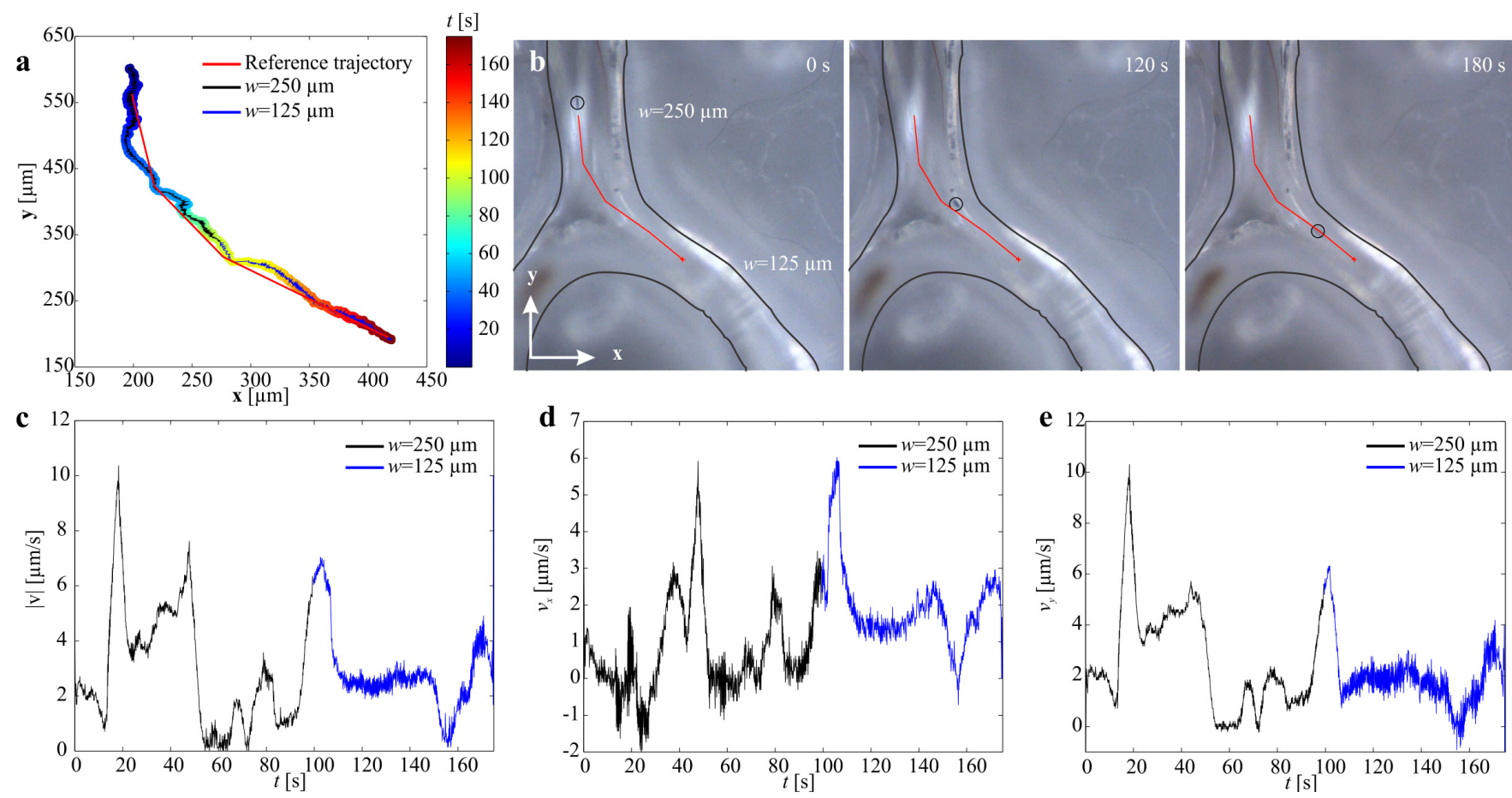

Fig. 6. Closed-loop control of a robotic sperm is achieved inside a channel along a reference trajectory at actuation frequency of $10 \mathrm{~Hz}$. (a) The robotic sperm swims in a relatively wide channel $(w=250 \mu \mathrm{m})$. (b) The robotic sperm reaches at the bifurcation point of the channel at time $t=100 \mathrm{~s}$. Flagellar propulsion is achieved along a reference trajectory between channels with width of $500 \mu \mathrm{m}$ and $250 \mu \mathrm{m}$. The black circle indicates the head of the robotic sperm, whereas the black lines show the edges of the channel. (c) The average swimming speed of the robotic sperm before and after the bifurcation point is $1.46 \pm 0.9 \mu \mathrm{m} / \mathrm{s}$ and $1.38 \pm 0.6 \mu \mathrm{m} / \mathrm{s}$, respectively. (d) The average swimming speeds of the robotic sperm along $\mathrm{x}$-axis before and after the bifurcation are measured as $0.42 \pm 0.6 \mu \mathrm{m} / \mathrm{s}$ and $0.94 \pm 0.5 \mu \mathrm{m} / \mathrm{s}$, respectively. (e) The average swimming speeds of the robotic sperm along $\mathbf{y}$-axis before and after the bifurcation are measured as $1.35 \pm 0.9 \mu \mathrm{m} / \mathrm{s}$ and $0.95 \pm 0.5 \mu \mathrm{m} / \mathrm{s}$, respectively. Please refer to the accompanying video.

speed of the robotic sperm before and after the bifurcation is $1.46 \pm 0.9 \mu \mathrm{m} / \mathrm{s}$ and $1.38 \pm 0.6 \mu \mathrm{m} / \mathrm{s}$, respectively [Figs. 6(c) and 6(e)]. This experiment is repeated using the same robotic sperm at actuation frequency of $5 \mathrm{~Hz}$, as shown in Figs. 7(a) and 7(b). The average speed of the robotic sperm is measured as $1.69 \pm 0.9 \mu \mathrm{m} / \mathrm{s}$ before the bifurcation point of the channel $(w=250 \mu \mathrm{m})$. The average speed of the robotic sperm past the bifurcation decreases to $0.82 \pm 0.8 \mu \mathrm{m} / \mathrm{s}$ for $w=125 \mu \mathrm{m}$ [Figs. 7(c) and 7(e)]. These control experiments show that the average speed of the robotic sperm decreases by $5.5 \%$ and $51.4 \%$ when it swims from the wide channel to the narrow channel, for actuation frequencies of $10 \mathrm{~Hz}$ and $5 \mathrm{~Hz}$, respectively. Please refer to the accompanying video.

\section{CONCLUSions AND Future Work}

This paper reports on the near surface effects on the flagellar propulsion of soft robotic sperms inside fluidic channel of varying width, under the influence of periodic magnetic fields. Our hydrodynamic model shows that the deformation of the beating tail is influenced by the proximity to a nearby surface due to the dependence of the normal and parallel drag on the distance to a nearby surface. The maximum amplitude of the propagating wave along the flexible tail decreases as the distance between the robotic sperm and a nearby wall is decreased. In addition, our model shows that the near surface effects can be mitigated using the actuation frequency and the precision angle of the robotic sperm. We also demonstrate point-to-point closed-loop control of robotic sperms along reference trajectories (with maximum steady-state error of $5.6 \mu \mathrm{m})$ inside a channel with bifurcations and varying width to experimentally examine the near surface effect on flagellar propulsion.

As part of future studies, we will investigate flagellar propulsion in a non-uniform flow-field inside fluidic microchips. It is likely that soft robotic sperm will be used to achieve targeted drug delivery in bodily fluids with nonuniform flow rates. Therefore, this investigation is essential to translate robotic sperms into in vivo experiments. In addition, soft robotic sperm samples are fabricated using polystyrene, and hence our electrospinning technique will be modified and a biodegradable polymer will be used.

\section{REFERENCES}

[1] J. J. Abbott, K. E. Peyer, M. C. Lagomarsino, L. Zhang, L. Dong, I. K. Kaliakatsos, and B. J. Nelson, "How should microrobots swim?," The International Journal of Robotics Research, vol. 28, no. 11-12, pp. 1434-1447, November 2009.

[2] S. Martel and M. Mohammadi, "Using a swarm of self-propelled natural microrobots in the form of flagellated bacteria to perform complex micro-assembly tasks," in Proceedings of The IEEE International Conference on Robotics and Automation (ICRA), pp. 500-505, Alaska, USA, May 2010.

[3] O. Felfoul, M. Mohammadi, S. Taherkhani, D. de Lanauze, Y. Z. Xu, D. Loghin, S. Essa, S. Jancik, D. Houle, M. Lafleur, L. Gaboury, M. Tabrizian, N. Kaou, M. Atkin, T. Vuong, G. Batist, N. Beauchemin, 

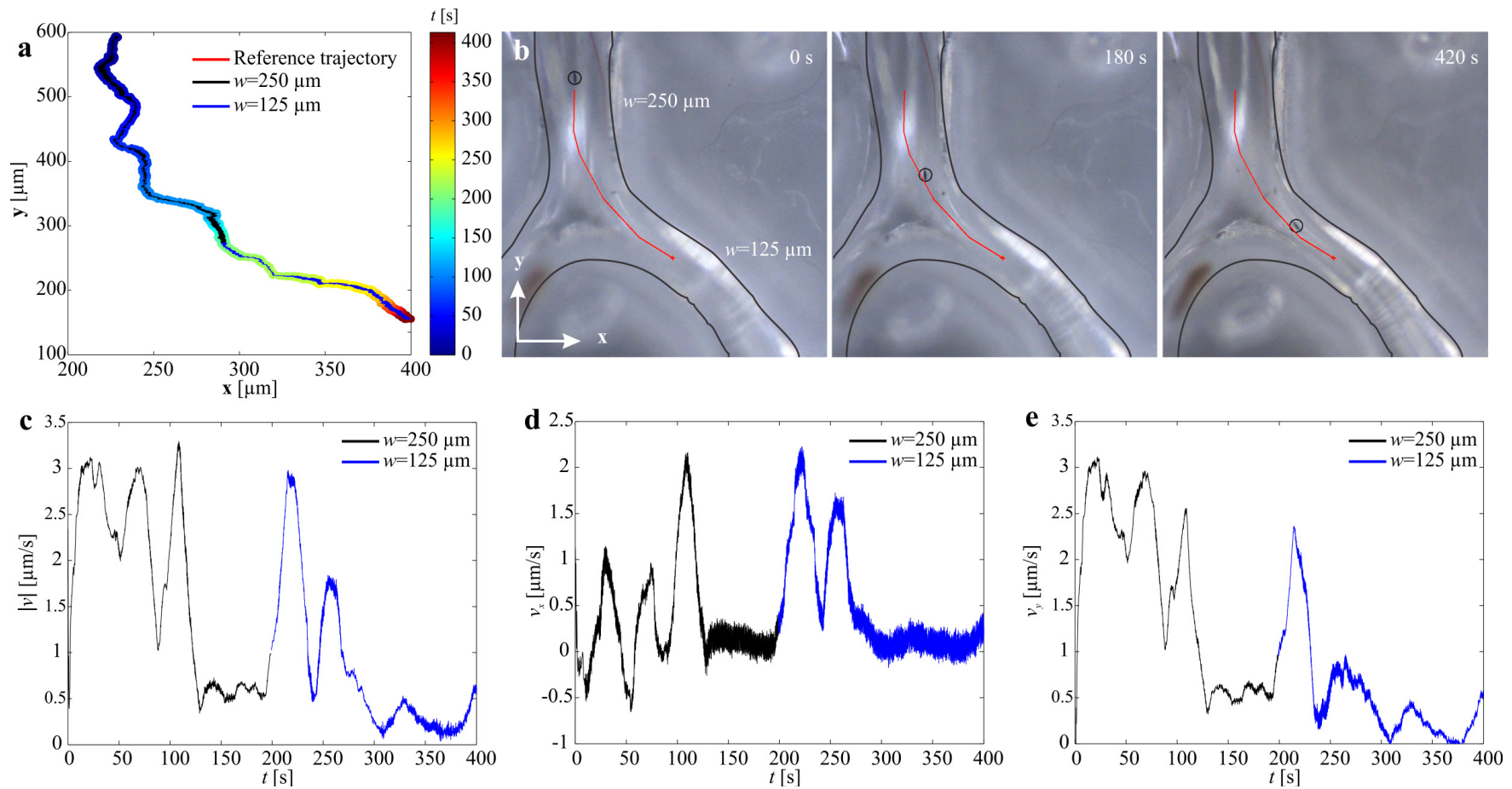

Fig. 7. Closed-loop control of a robotic sperm is achieved inside a channel along a reference trajectory at actuation frequency of $5 \mathrm{~Hz}$. (a) The robotic sperm swims in a relatively wide channel $(w=250 \mu \mathrm{m})$ and reaches at the bifurcation point of the channel at time $t=100$ seconds. (b) Flagellar propulsion is achieved along a reference trajectory between channels with width of $500 \mu \mathrm{m}$ and $250 \mu \mathrm{m}$. The black circle indicates the head of the robotic sperm, whereas the black lines show the edges of the channel. (c) The average swimming speed of the robotic sperm before and after the bifurcation point is $1.69 \pm 0.9 \mu \mathrm{m} / \mathrm{s}$ and $0.82 \pm 0.8 \mu \mathrm{m} / \mathrm{s}$, respectively. (d) The average swimming speeds of the robotic sperm along $\mathbf{x}$-axis before and after the bifurcation are measured as $0.32 \pm 0.5 \mu \mathrm{m} / \mathrm{s}$ and $0.52 \pm 0.6 \mu \mathrm{m} / \mathrm{s}$, respectively. (e) The average swimming speeds of the robotic sperm along $\mathbf{y}$-axis before and after the bifurcation are measured as $1.58 \pm 0.9 \mu \mathrm{m} / \mathrm{s}$ and $0.54 \pm 0.5 \mu \mathrm{m} / \mathrm{s}$, respectively. Please refer to the accompanying video.

D. Radzioch, and S. Martel, "Magneto-aerotactic bacteria deliver drug-containing nanoliposomes to tumour hypoxic regions", Nature Nanotechnology, August 2016.

[4] A. Servant, F. Qiu, M. Mazza, K. Kostarelos, and B. J. Nelson, "Controlled in vivo swimming of a swarm of bacteria-like microrobotic flagella," Advanced Materials, vol. 27, no. 19, pp. 2981-2988, April 2015.

[5] S. K. Srivastava, M. M.-Sánchez, B. Koch, and O. G. Schmidt, "Medibots: dual-action biogenic microdaggers for single-cell surgery and drug release," Advanced Materials, vol. 28, no. 5, pp. 832-837, November 2015.

[6] H.-W. Huang, Q. Chao, M. S. Sakar, and B. J. Nelson, "Optimization of tail geometry for the propulsion of soft microrobots," IEEE Robotics and Automation Letters, vol. 2, no. 2, pp. 727-732, January 2017.

[7] T. Xu, C. Vong, B. Wang, L. Liu, X. Wu, and L. Zhang, "Rotating soft-tail millimeter-scaled swimmers with superhydrophilic or superhydrophobic surfaces," in Proceedings of the IEEE International Conference on Biomedical Robotics and Biomechatronics (BioRob), pp. 502-507, Singapore, July 2016.

[8] O. S. Pak, W. Gao, J. Wang, and E. Lauga, "High-speed propulsion of flexible nanowire motors: Theory and experiments," Soft Matter, vol. 7, pp. 8169-8181, July 2011.

[9] B. Jang, E. Gutman, N. Stucki, B. F. Seitz, P. D. Wendel-García, T. Newton, J. Pokki, O. Ergeneman, S. Pané, Y. Or, and B. J. Nelson, "Undulatory locomotion of magnetic multilink nanoswimmers," Nano Letters, vol. 15, no. 7, pp. 4829-4833, June 2015.

[10] K. K. Coti, M. E. Belowich, M. Liong, M. W. Ambrogio, Y. A. Lau, H. A. Khatib, J. I. Zink, N. M. Khashab, and J. F. Stoddart, "Mechanised nanoparticles for drug delivery," Nanoscale, vol. 1, no. 16, pp. 16-39, September 2009

[11] S. Campuzano, D. Kagan, J. Orozco, and J. Wang, "Motion-Driven sensing and biosensing using electrochemically propelled nanomotors," Analyst, 136, 4621-4630, September 2011.

[12] J. Liu, J. Wen, Z. Zhang, H. Liu, and Y. Sun, "Voyage inside the cell: microsystems and nanoengineering for intracellular measurement and manipulation," Microsystems \& Nanoengineering, vol. 1, no. 15020, September 2015.

[13] B. E.-Fernández de Ávila, P. Angsantikul, J. Li, M. A. Lopez-Ramirez, D. E. Ramrez-Herrera, S. Thamphiwatana, C. Chen, J. Delezuk, R. Samakapiruk, V. Ramez, L. Zhang, and J. Wang, "Micromotor-enabled active drug delivery for in vivo treatment of stomach infection," Nature Communications, vol. 8, no. 272, August 2017.

[14] D. Ahmed, C. Dillinger, A. Hong, and B. J. Nelson, "Artificial acoustomagnetic soft microswimmers," Advanced Materials Technologies, vol. 2, no. 7 (1700050), May 2017.

[15] E. M. Purcell, "Life at low reynolds number," American Journal of Physics, vol. 45, no. 1, pp. 3-11, June 1977.

[16] C. H. Wiggins and R. E. Goldstein, "Flexive and propulsive dynamics of elastica at low reynolds number," Physical Review Letters, vol. 80, no. 17 , pp. 3879-3882, April 1998.

[17] R. Dreyfus, J. Baudry, M. L. Roper, M. Fermigier, H. A. Stone, and J. Bibette, "Microscopic artificial swimmers." Nature, vol. 437, October 2005.

[18] C. Brennen and H. Winet, "Fluid mechanics of propulsion by cilia and flagella," Annual Review of Fluid Mechanics, vol. 9, pp. 339398, January 1977.

[19] I. S. M. Khalil, A. F. Tabak, A. Klingner, and M. Sitti, "Magnetic propulsion of robotic sperms at low-Reynolds number," Applied Physics Letters, vol. 109 (033701), July 2016.

[20] I. S. M. Khalil, A. F. Tabak, Y. Hamed, M. Elwi, M. Tawakol, A. Klingner, and M. Sitti, "Swimming back and forth using planar flagellar propulsion at low Reynolds numbers," Advanced Science, 1700461, December 2017.

[21] I. S. M. Khalil, H. C. Dijkslag, L. Abelmann, and S. Misra, "MagnetoSperm: A microrobot that navigates using weak magnetic fields," Applied Physics Letters, vol. 104 (223701), June 2014. 